Diabetologia 9, 396-399 (1973)

(C) by Springer-Verlag 1973

\title{
Release of Large Amounts of Noradrenaline from the Isolated Perfused Canine Pancreas during Glucose Deprivation
}

\author{
N.J. Christensen and J. Iversen \\ Second University Clinic of Internal Medicine, Kommunehospitalet, 8000 Aarhus, Denmark. \\ Received: January 25, 1973, in revised form: May 24, 1973
}

\begin{abstract}
Summary. Six fasting male mongrels served as pancreas donors. The pancreas was perfused without recirculation with a synthetic medium. The noradrenaline and adrenaline concentration in the efflux perfusate was determined by a double-isotope derivative technique. 1 . The noradrenaline concentration in the efflux perfusate rose considerably (from $0.25 \mathrm{ng} / \mathrm{ml}$ to $10.0 \mathrm{ng} / \mathrm{ml}$ ), when the pancreas was perfused with a glucose deprived perfusing medium. The concentration rose almost linearly with time. After the addition of very small amounts of glucose $(2 \mathrm{mg} / 100 \mathrm{mI})$ to the perfusing medium there was a considerable decrease in catecholamine concentration and a further decrease with higher glucose concentrations. 2. No
\end{abstract}

change in catecholamine concentration in the efflux perfusate was observed if the pancreas was perfused with a high glucose concentration during the whole experiment. 3 . Glucagon release was also high during perfusion with a glucose deprived solution while insulin release was low. These experiments raise the question whether an increased catecholamine release may, at least partially, be responsible for the change in insulin and glucagon secretion during glucose deprivation.

Key words: Isolated perfused pancreas, catecholamine release, insulin release, glucagon release, glucose, autonomic nervous system.
In a series of studies designed to evaluate catecholamine release and uptake in the splanchnic area, it was found that the isolated perfused canine pancreas released considerable amounts of noradrenaline during perfusion with a glucose deprived solution. In view of the known effect of catecholamines on insulin and glucagon release it was considered of interest to study these phenomena in more detail.

\section{Material and Methods}

Six overnight fasted male mongrels weighing 18 $25 \mathrm{~kg}$ served as pancreas donors. The operative procedure for isolation of the canine pancreas and the perfusion system has been described in detail elsewhere [1]. The pancreas was perfused without recirculation, with a synthetic medium consisting of a Krebs-Ringer solution which was adjusted to the electrolyte concentrations found in dog plasma, containing $4 \%$ dex$\operatorname{tran}(\mathrm{Mw} 75000), 0.2 \%$ bovine albumin, glutamate, fumarate and pyruvate, each at a concentration of 5 $\mathrm{mM}$. The perfusion flow was $18-20 \mathrm{ml} / \mathrm{min}$ (approximately $0.25 \mathrm{ml} / \mathrm{min} / \mathrm{g}$ of pancreas, wet weight) and the perfusion pressure was $30-40 \mathrm{~mm} \mathrm{Hg}$. Both parameters were constant during the experimental period. The oxygen uptake of the isolated pancreas was 0.005 $\mathrm{ml} / \mathrm{g} / \mathrm{min}[1]$.

The noradrenaline concentration and the adrenaline concentration or the sum of these two catecholamines (PCA) in the efflux perfusate was determined by the double-isotope derivative assay described by Engelman \& Portnoy [2], except for minor modifications. Reproducibility and recovery experiments have been pres- ented elsewhere $[3,4]$. In most cases the sum of noradrenaline and adrenaline was measured because preliminary experiments showed that the catecholamines released from the pancreas consisted mainly of noradrenaline (approximately $90 \%$ ).

Pancreatic glucagon and insulin were determined by radioimmunoassay employing wick-chromatography [5] with minor modifications [6].

The concentration of noradrenaline in the intact resting dog is $0.10-0.30 \mathrm{ng} / \mathrm{ml}$ in peripheral venous plasma. The adrenaline concentration is approximately $0.05 \mathrm{ng} / \mathrm{ml}$. In the fasting dog plasma insulin is approximately 5 to $10 \mu \mathrm{U} / \mathrm{ml}$ and plasma glucagon 80 to 100 $\mathrm{pg} / \mathrm{ml}$, (pancreatic vein glucagon $3-400 \mathrm{pg} / \mathrm{ml}$ ).

\section{Results}

Table 1 shows the results of two pilot experiments. In both cases the catecholamine concentration in the efflux perfusate was very high when the perfusing

Table 1. Glucose and Catecholamines (PCA) in Efflux Perfusate in Two Preliminary Experiments

\begin{tabular}{lcc}
\hline $\begin{array}{l}\text { Exp. } \\
\text { no. }\end{array}$ & Glucose & PCA \\
mg/ $100 \mathrm{ml}$ & $\mathrm{ng} / \mathrm{ml}$ \\
\hline 1$)$ & 0 & 0.75 \\
& 150 & 0.08 \\
$2)$ & 150 & 0.08 \\
& 0 & 2.25 \\
& 5 & 0.66 \\
& 0 & 5.22 \\
& 5 & 1.40 \\
\hline
\end{tabular}


Table 2: Glucose, Catecholamines (POA), Glucagon and Insulin in Efflux Perfusate

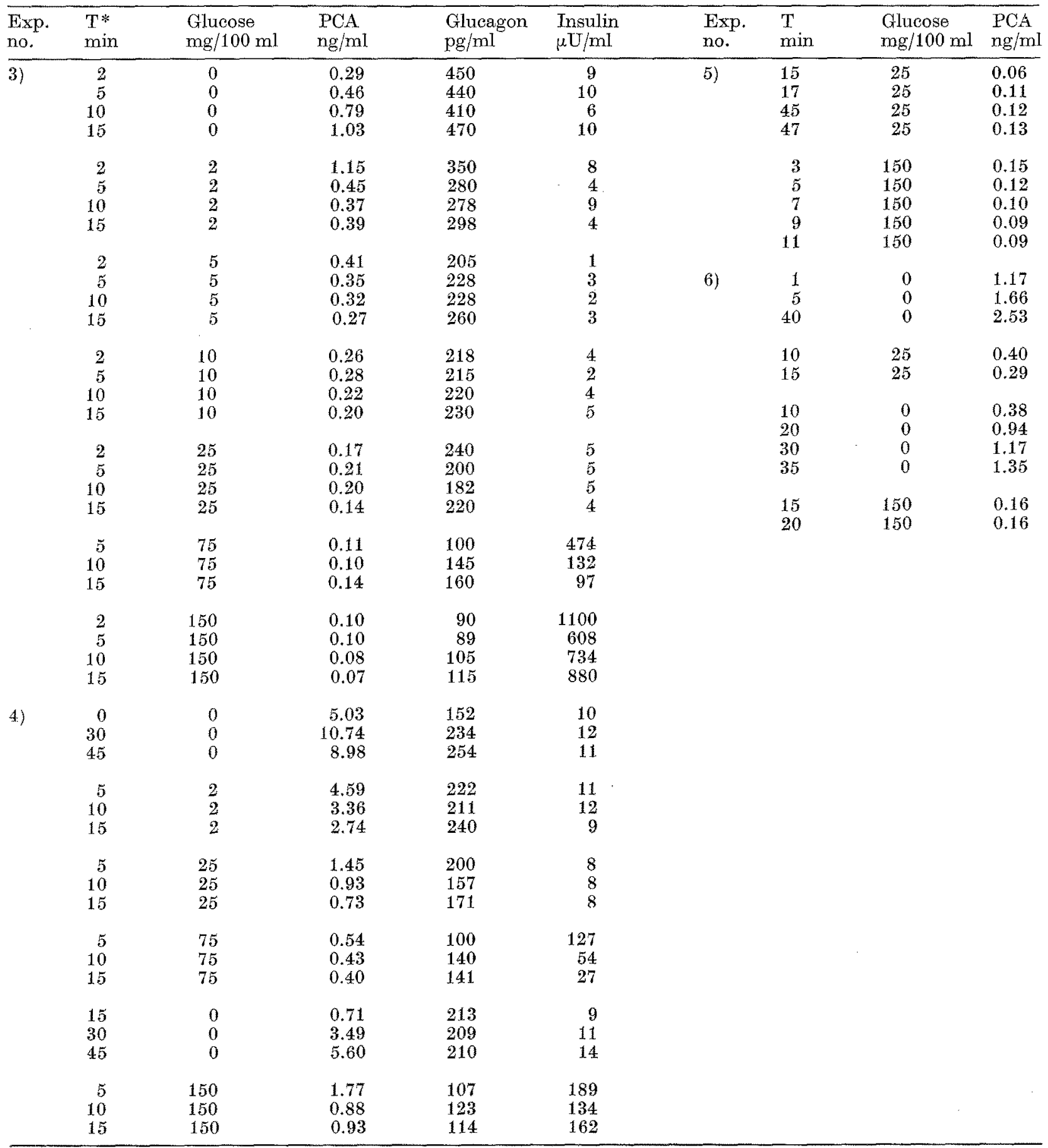

* T denotes the time in min within the periods of perfusion with a given glucose concentration. Before sampling from each of the four experiments the pancreas was perfused for an equilibration period of $10-20 \mathrm{~min}$ with $0 \mathrm{mg} / 100 \mathrm{ml}$ glueose (Exp. no. 3,4 and 6) and $25 \mathrm{mg} / 100 \mathrm{ml}$ (Exp. no. 5).

medium was deprived of glucose and there was a considerable decrease in the concentration after addition of glucose to the perfusing fluid.

Table 2 shows the results of four additional experiments which were designed to study in more detail the relationship between different glucose concentrations in the perfusion medium and the release of catecholamines from the pancreas.

In three of the four cases the pancreas was perfused initially with a glucose deprived perfusing medium. 
The catecholamine concentration in the efflux perfusate was again very high in these experiments. The concentration rose almost linearly with time, except in the initial part of experiment no. 4 .

After the addition of very small amounts of glucose, i.e. $2 \mathrm{mg} / 100 \mathrm{ml}$ to the perfusing medium, there was a pronounced decrease in the catecholamine concentration and a further decrease with a stepwise increase in glucose concentration.

In two of the experiments perfusion with no glucose in the medium was repeated later in the experiment (exp. no 4 and 6) and it can be seen that the concentration of the catecholamines in the efflux rose again. In exp. no 6 a similar increase per unit of time was observed in both situations.

In exp. no 5 (Table 2) the perfusing medium contained glucose $(25$ and $150 \mathrm{mg} / 100 \mathrm{ml}$ ) during the whole experiment. The catecholamine concentrations in the efflux remained low.

The plasma catecholamine concentration in the perfusate at zero glucose was significantly higher than the catecholamine content in the perfusate at any succeeding higher glucose concentration (paired t-test, $\mathrm{p}$ less than 0.01 ).

Table 2 also presents the glucagon and insulin values. During perfusion with a glucose deprived perfusion medium glucagon release was high and insulin release was low. This pattern of release was reversed during a stepwise increase in the glucose concentration to $150 \mathrm{mg} / 100 \mathrm{ml}$, with inhibition of glucagon and stimulation of insulin output, as previously described for this preparation [6].

\section{Discussion}

The present study shows that very large amounts of noradrenaline are released from the isolated perfused canine pancreas during glucose deprivation. The possibility that the release of catecholamines could be due to the preparation of the pancreas, e.g. cutting the nerves, is unlikely since low levels and no increase in catecholamines were observed when the pancreas was perfused with a glucose concentration of 25 to $150 \mathrm{mg} / 100$ $\mathrm{ml}$ during the whole experiment. Furthermore, the fact that large amounts of catecholamines were released later in the experiments, when perfusion with no glucose in the perfusing medium was repeated, seems to exclude this possibility. The catecholamine concentration was higher during perfusion with a glucose deprived solution when this was performed in the beginning of the experiment (exp. no 4 and 6). However, the rise per unit of time was the same (exp. no 6). This finding may be explained by the fact that sampling was not started until after an initial equilibration period of 10 to $20 \mathrm{~min}$ perfusion (Table 2). In this situation the cells have been without glucose for a longer period of time.
It is difficult from the present experiments to state a glucose concentration which will suppress catecholamine release maximally, because the period of perfusion at a given glucose concentration was probably not always of a sufficient duration to obtain steady state catecholamine concentrations. It is clear, however, that the major effect was induced by rather low glucose concentrations in the range 2 to $25 \mathrm{mg} / 100 \mathrm{ml}$.

The increased release of noradrenaline from the isolated pancreas is probably mainly due to an increase in noradrenaline secretion (from the postganglionic nerve fibres) and not due to an inhibition of neuronal re-uptake alone. Although administration of membrane pump blockers increases noradrenaline output from various organs, they do not lead to such a massive change as observed in the present study [7]. In experiments in the intact dog where the basal plasma noradrenaline concentration is approximately similar to that found in the effluent from the pancreas when the perfusate contains $75 \mathrm{mg} / 100 \mathrm{ml}$ of glucose, administration of phentolamine, which inhibits re-uptake, will only increase catecholamine concentration two-fold (to $0.30 \mathrm{ng} / \mathrm{ml}$ ) (unpublished observation).

Wakade and Furchgott [8] studied the metabolic requirements for the uptake and the storage of norepinephrine by the isolated left atrium of the guinea pig. Uptake and retention of noradrenaline were not altered by inhibition of oxidative metabolism or by inhibition of glycolysis. Simultaneous inhibition of oxidative metabolism and glycolysis markedly decreased net uptake and depleted endogenous noradrenaline stores. Rubin [9], who worked with the perfused adrenal gland, observed no increase in catecholamine secretion during glucose deprivation alone. Glucose deprivation combined with anoxia caused an increase in the spontaneous catecholamine output from the gland. Anoxia and glucose deprivation depressed the secretory response to acetylcholine and calcium $[9,10,11]$. The mechanism by which glucose deprivation alone leads to such massive changes in noradrenaline output in the canine pancreas is obscure at the moment and deserves further investigation. In a recent abstract Malik and McGiff [12] reported that the constrictor response to postganglionic nerve stimulation in the mesenteric vessels of the rat was markedly potentiated during glucose deprivation.

The response of noradrenaline release to glucose deprivation in the in vitro canine pancreas appears similar to that of glucagon release and contrary to that of insulin. Additional experiments are necessary to determine whether an increased catecholamine release may, at least partially, be responsible for the changes in insulin and glucagon secretion during glucose deprivation.

The in vitro pancreas perfusion technique is a commonly used preparation which may be suitable for the study of catecholamine release and uptake during various conditions. 


\section{References}

1. Iversen, J., Miles, D.W.: Evidence for a feedback inhibition of insulin on insulin secretion in the isolated, perfused canine pancreas. Diabetes 20, 1-9 (1971)

2. Engelman, K., Portnoy, B.: A sensitive double isotope derivative assay for norepinephrine and epinephrine. Circulat. Res. 26, 53-57 (1970)

3. Christensen, N.J.: Plasma catecholamines in longterm diabetics with and without neuropathy and in hypophysectomized subjets. J. clin. Invest. 51, 779787 (1972)

4. Christensen, N.J.: Increased levels of plasma noradrenaline in hypothyroidism. J. clin. Endocrin. 35, 359$363(1972)$

5. Ørskov, H.: Wick-chromatography for the immunoassay of insulin. Scand. J. clin. Lab. Invest. 20, 297$304(1967)$

6. Iversen, J.: Secretion of glucagon from the isolated perfused canine pancreas. J. clin. Invest. 50, 2123$2137(1971)$

7. Häggendal, J.: In : New aspects of storage and release mechanisms of catecholamines, Page 100. H.J. Schümann and G. Kroneberg (eds.) New York 1970
8. Wakade, A.R., Furchgott, R.F.: Metabolic requirements for the uptake and storage of norepinephrine by the isolated left atrium of the guinea pig. J. Pharmacol. exp. Ther. 163, 123-135 (1968)

9. Rubin, R.P.: The metabolic requirements for catecholamine release from the adrenal medulla. J. Physiol. 202, 197-209 (1969)

10. Rubin, R.P.: The role of energy metabolism in calcium evoked secretion from the adrenal medulla. J. Physiol. (Lond.) 206, 181-192 (1970)

11. Kirshner, N., Smith, W.J.: Metabolic requirements for secretion from the adrenal medulla. Life Sciences 8, 799-803 (1969)

12. Malik, K.U., McGiff, J.C. : Role of glucose in adrenergie transmission of rat perfused mesenteric arteries. Clin. Res. 20, 719 (abstract) (1972)

Dr. Johan Iversen Kommunehospitalet Second University Clinic of Internal Medicine DK-8000 Aarhus C Denmark 